\title{
Materials synthesis and crystallography at extreme pressure-temperature conditions
}

\author{
Natalia Dubrovinskaia ${ }^{1}$, Leonid Dubrovinsky ${ }^{2}$ \\ ${ }^{1}$ Laboratory of Crystallography, Universität Bayreuth, Bayreuth, Germany; \\ ${ }^{2}$ Bayerisches Geoinstitut, Universität Bayreuth, Bayreuth, Germany;
}

\section{Natalia.Dubrovinskaia@uni-bayreuth.de}

During last decades, the impact of high-pressure studies on fundamental physics, chemistry, and Earth and planetary sciences, has been enormous. Modern science and technology rely on the vital knowledge of matter which is provided by crystallographic investigations. The most reliable information about crystal structures of solids and their response to alterations of pressure and temperature is obtained from single-crystal diffraction experiments. Advances in diamond anvil cell (DAC) techniques, designs of double-stage DACs, and in modern X-ray instrumentation and synchrotron facilities have enabled structural research at multimegabar pressures.

We have developed a methodology for performing single-crystal X-ray diffraction experiments in double-side laser-heated DACs and demonstrated that it allows the crystal structure solution and refinement, as well as accurate determination of thermal equations of state above $200 \mathrm{GPa}$ at temperatures of thousands of degrees. Application of this methodology resulted in discoveries of novel compounds with unusual chemical compositions and crystal structures, uncommon crystal chemistry and physical properties. Perspectives of materials synthesis and crystallography at extreme conditions will be outlined.

Keywords: high-pressure crystallography, single-crystal XRD, DAC, LVP 